\title{
Color Image Enhancement using Correlated Intensity and Saturation Adjustments
}

To appear in the Journal of Modern Optics

Vol. 00, No. 00, 00 Month 20XX, 1-12

\author{
Ngaiming Kwok ${ }^{\mathrm{a} *}$, Haiyan $\mathrm{Shi}^{\mathrm{b}}$, Gu Fang ${ }^{\mathrm{c}}$, Quang $\mathrm{Ha}^{\mathrm{d}}$, Ying-Hao Yu${ }^{\mathrm{e}}$, Tonghai $\mathrm{Wu}^{\mathrm{f} *}$ \\ Huaizhong $\mathrm{Li}^{\mathrm{g}}$ and Thai Nguyen ${ }^{\mathrm{a}}$ \\ ${ }^{a}$ School of Mechanical and Manufacturing Engineering, The University of New South Wales, Australia \\ ${ }^{b}$ School of Computer Science and Technology, Shaoxing University, Shaoxing, Zhejiang, China \\ ${ }^{c}$ School of Computing, Engineering and Mathematics, University of Western Sydney, Australia \\ ${ }^{d}$ School of Electrical, Mechanical and Mechatronic Systems, University of Technology Sydney, Australia \\ ${ }^{e}$ Department of Electrical Engineering, National Chung Cheng University, Taiwan \\ ${ }^{f}$ Key Laboratory of Modern Design and Rotor Bearing System of Ministry, Xi'an Jiaotong University, \\ Xi'an, Shaanxi, China \\ ${ }^{g}$ Griffith School of Engineering, Griffith University, Australia
}

(Received 00 Month 20XX; final version received 00 Month 20XX)

\begin{abstract}
The enhancement of digital color images needs to be performed in accordance with human perceptions in terms of hue, saturation and intensity attributes instead of improving only the contrast. Two approaches were developed in this work which use a correlated adjustment mechanism incorporating intensity and saturation attributes and provide contrast and saturation enhancements together with brightness consistency. In these algorithms, object edges are emphasized for contrast, and image saturation is increased by boosting the salient regions. Furthermore, intensity and saturation enhancements are carried out in a lattice structure where adjustments are made inter-related for better performance. Experiments were conducted with benchmark and real-world images. Results had shown improvements in image qualities both qualitatively and quantitatively.
\end{abstract}

Keywords: color image; contrast enhancement; saturation enhancement; color enhancement; brightness preservation

\section{Introduction}

Digital color image technologies have been used in a large number of engineering applications. For instance, these techniques can be employed in remote sensing of the land [1], and the inspection of wear debris [2] for machine condition monitoring. Other examples include the restoration of ancient paintings [3] and use in robotic control [4]. In order to ensure satisfactory performances, it is required to enhance the image quality which still remains as one of the challenging and demanding tasks.

The image quality is closely related to human perception attributes in hue, saturation and intensity. Hue can be improved against illumination color cast by means of color correction [5]. The contrast can be improved by manipulating the intensity distribution [6] to highlight the objects captured in the image. Further, to emphasizing color and intensity attributes, improvements in saturation could greatly increase visual comfort [7]. A method was suggested in [8] to directly adjust the saturation in the CIExy color space. Moreover, techniques that detect the sky region in

*Corresponding author. Ngaiming Kwok, nmkwok@unsw.edu.au, Tonghai Wu, tonghaiwu@mail.xjtu.edu.cn. 
natural scenes [9] and use fuzzy theory [10] to adjust image saturation had also been developed.

Methods for image quality enhancement encompass several categories of approaches. One of the categories integrates intensity and saturation adjustments to accomplish the enhancement goal [11]. A further refinement was made that incorporates the correlation between intensity and saturation for enhancement purpose [12]. Their primary focus is to increase the contrast and saturation of the image where object details are made distinctive and colors are made more vivid.

This work aims to enhance image qualities in terms of both the contrast and saturation. It begins with the examination of the principle that integrates intensity and saturation manipulation. A simple method for efficiency is first proposed and an improvement on effectiveness is further developed. These methods employ a lattice structure of scaled intensity and saturation adjustment factors to improve the image quality corresponding to human perceptions.

The rest of the paper is organized as follows. In Section 2, related works in integrated adjustments are reviewed. The proposed methods with lattice structure based image quality enhancement are presented in Section 3. Section 4 describes experiments conducted and evaluates the results. Finally, a conclusion is drawn in Section 5 .

\section{Related Work}

Among the methods that address the enhancement of digital color images, there is a class of approaches that are concerned with the simultaneous improvement on contrast and saturation [12]. These include a method that combines intensity adjustment with input-output image saturation ratio as suggested in [13]. This method was later simplified which use only a power law adjustment on the saturation ratio [14]. Furthermore, an integration of differential histogram and weighted intensity and saturation adjustment was proposed independently [15]. An introduction of these methods is presented below.

\subsection{Intensity Adjustment by Intensity and Saturation Feedback}

Given a color image, the intensity component is first extracted from converting the red green blue (RGB) to the hue saturation intensity (HSI) color space [16], and then adjusted for improved output image contrast [11]. Let the input intensity and saturation be denoted by $I_{\text {in }}$ and $S_{i n}$, the enhanced intensity $I_{e n h}$ is obtained from

$$
I_{e n h}(u, v)=I_{i n}(u, v)+k_{1}\left(I_{i n}(u, v)-\bar{I}_{i n}(u, v)\right)+k_{2}\left(S_{i n}(u, v)-\bar{S}_{i n}(u, v)\right),
$$

where $(u, v)$ is the pixel coordinate. Scale factors $k_{1}$ and $k_{2}$ are responsible for the strength of enhancements based on intensity and saturation. Furthermore, the local averages $\bar{I}_{i n}(u, v)$ and $\bar{S}_{i n}(u, v)$ are derived from averaging the pixel magnitudes in a square patch $\boldsymbol{\Omega}$ of size $W \times W$ centered at coordinate $(u, v)$.

The differences between the input attributes and their local averages, namely,

$$
\begin{aligned}
\Delta I(u, v) & =I_{i n}(u, v)-\bar{I}_{i n}(u, v), \\
\Delta S(u, v) & =S_{i n}(u, v)-\bar{S}_{i n}(u, v),
\end{aligned}
$$

extract the edges around objects where their contrasts are to be highlighted. This process is similar in concept to the unsharp masking filter [17] with an added contribution from the saturation characteristics. Note that in this approach, called intensity adjustment by intensity and saturation (IAIS), only the output intensity is enhanced but not the output saturation. 


\subsection{Intensity Adjustment by Intensity and Saturation Correlated Feedback}

In order to increase the effectiveness, another method uses the correlation $\rho(u, v)$ between intensity and saturation to adjust the output intensity [12], called intensity adjustment by intensity and saturation correlate (IAISC). The modification from IAIS is given as

$$
I_{e n h}(u, v)=I_{i n}(u, v)+k_{1}\left(I_{i n}(u, v)-\bar{I}_{i n}(u, v)\right)+k_{2} \rho(u, v)\left(S_{i n}(u, v)-\bar{S}_{i n}(u, v)\right),
$$

where the correlation is

$$
\rho(u, v)=\frac{\sum_{\Omega} \Delta I(u, v) \Delta S(u, v)}{\sqrt{\sum_{\Omega} \Delta I^{2}(u, v) \sum_{\Omega} \Delta S^{2}(u, v)}} .
$$

The manipulated intensity $I_{e n h}(u, v)$ then, together with the original input image hue and saturation, forms the output enhanced image. This approach also enhances only the output image intensity contrast but not the output saturation.

\subsection{Other Approaches}

In the work reported in [14], image enhancement was accomplished by using power law corrections on both intensity and saturation. Unlike other methods, the adjustment did not include the correlation between intensity and saturation. The modified intensity and saturation components are

$$
\begin{gathered}
I_{e n h}(u, v)=2 I_{i n}(u, v)-\bar{I}_{i n}(u, v)+\Delta \hat{I}(u, v), \\
S_{e n h}(u, v)=2 S_{i n}(u, v)-\bar{S}_{i n}(u, v)+\Delta \hat{S}(u, v),
\end{gathered}
$$

where variables $\Delta \hat{I}(u, v)$ and $\Delta \hat{S}(u, v)$ are the edge features obtained from the associated power law correction. That is

$$
\begin{aligned}
\Delta \hat{I}(u, v) & =\left(I_{i n}(u, v)-\bar{I}_{i n}(u, v)\right)^{\gamma_{I}} \\
\Delta \hat{S}(u, v) & =\left(S_{i n}(u, v)-\bar{S}_{i n}(u, v)\right)^{\gamma_{S}}
\end{aligned}
$$

where $\gamma_{I}$ and $\gamma_{S}$ are the power law coefficients.

The idea of simultaneous use of intensity and saturation attributes to improve image quality had been combined with histogram equalization [15]. Two differential histograms were first constructed for intensity and saturation while the latter was modified by the intensity-saturation correlation. The second stage generated a weighted sum of those two histograms for overall equalization on the intensity. That is

$$
h(i)=\alpha h_{I}(i)+(1-\alpha) h_{S}(i), 0<\alpha<1,
$$

where $h(i)$ is the weighted histogram with respect to $i$-th intensity index, subscripts $I$ and $S$ denote histograms of intensity and saturation, and $\alpha$ is the weighting factor.

\subsection{Motivation}

The use of both intensity and saturation information for image quality improvement is an attractive idea and satisfactory results had been given by a number of algorithms. However, some simple methods [11][12] can be further improved for better performance, while complicated algorithms 
[14][15] can also be made more efficient. For instance, the construction of differential histograms, raising exponents, calculation of the correlation are computationally inefficient and have to be compromised between efficiency and effectiveness.

\section{Intensity and Saturation Correlated Adjustment}

In order to improve the quality of a color image in terms of its contrast and saturation, possible alternatives are examined first. Then improvements are suggested and consolidated into two algorithms described below.

Consider the input color image given as $\mathcal{F}_{\text {in }}(u, v)=F_{\text {in }}\{R, G, B\}(u, v)$, each pixel contains the three primary tri-stimuli RGB color channels. Let the input color image be converted to the HSI color space [16] in hue $H_{i n}(u, v)$, saturation $S_{i n}(u, v)$ and intensity $I_{i n}(u, v)$ components.

As motivated by the algorithm reported in [11], a simplified algorithm called intensity and saturation adjustment by intensity and saturation (ISAIS) is proposed. Adjustments on intensity and saturation are now given by

$$
\begin{gathered}
I_{e n h}(u, v)=I_{i n}(u, v)+k_{1} \Delta I(u, v), \\
S_{e n h}(u, v)=S_{i n}(u, v)+k_{2}|\Delta S(u, v)|,
\end{gathered}
$$

where $k_{1}, k_{2}$ are gain factors. The addition of $\Delta I(u, v)$ contributes to the increase in contrast. Since $\Delta S(u, v)$ could result in negative values and decreases the output saturation, the first modification is to limit its changes to increments thus an absolute function operator is included. On the other hand, adjustments in intensity have to incorporate both positive and negative changes for local contrasts enhancement, therefore, no limits on the change polarity are needed. Furthermore, intensity and saturation are manipulated independently.

A further refinement, called intensity and saturation adjustment by intensity and saturation correlation (ISAISC), is formulated. As suggested in [12], the adjustments on intensity and saturation both involve the correlation between them. However, the constraint on saturation adjustment and correlation to positive values are imposed. Then we have

$$
\begin{gathered}
I_{e n h}(u, v)=I_{i n}(u, v)+k_{1} \Delta I(u, v)+k_{2} \rho(u, v) \Delta S(u, v), \\
S_{e n h}(u, v)=S_{i n}(u, v)+k_{3} \Delta I(u, v)+k_{4}|\rho(u, v) \Delta S(u, v)|,
\end{gathered}
$$

where $k_{1}$ to $k_{4}$ are scale factors controlling the strengths of adjustment. A block diagram of this approach is depicted in Figure 1.

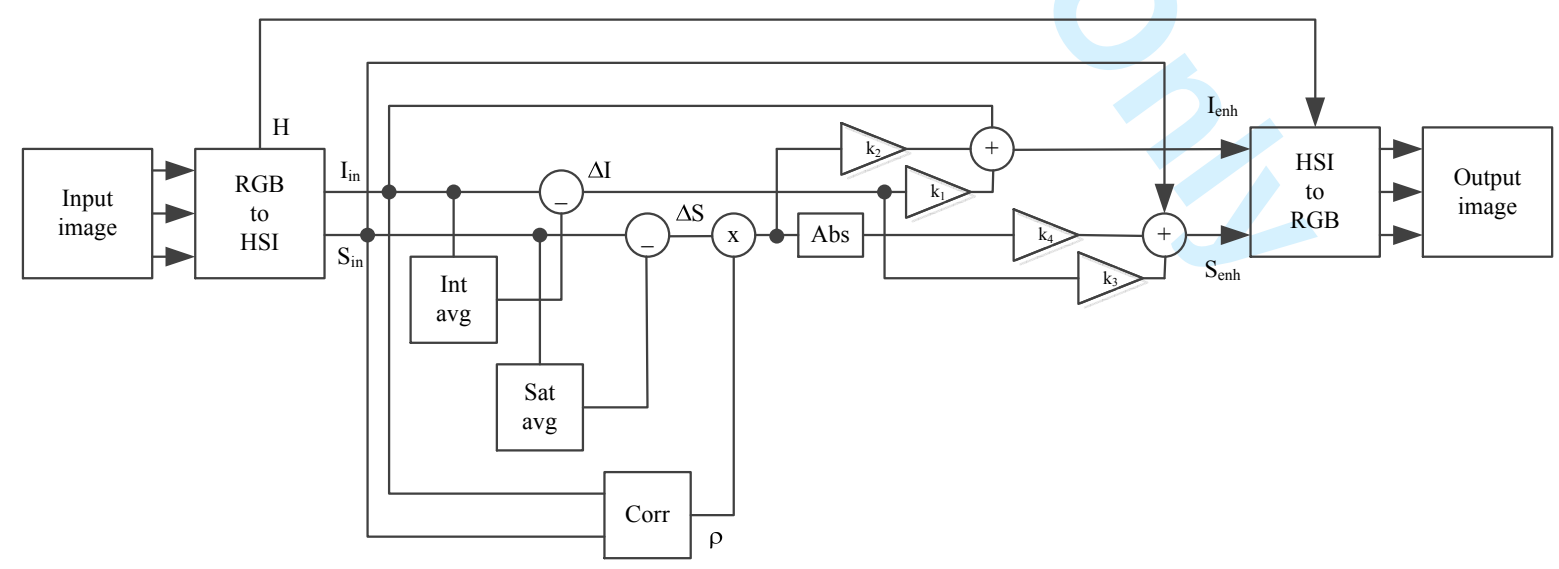

Figure 1. Block diagram of the ISAISC system. 


\section{Experiments}

Experiments were conducted to demonstrate the performance of the proposed approaches both qualitatively and quantitatively against a set of criteria. A collection of 120 color images were used. These include 20 publicly available images from http://r0k.us/graphics/kodak/. The rest are images of natural scenery taken under different environmental conditions such as clear day or poor conditions with overcast weather. The image sizes are $480 \times 360$ width-by-height and the images are delivered in JPG files of 8-bit data format in each RGB color channel. Comparisons are made between the input image, outputs from conventional uniform histogram equalization (UNFHE) [16], median-mean sub-image clipped histogram equalization (MMSICHE) [18], smoothed histogram equalization (SMHEQ) [19], IAIS [11], IAISC [12], ISAIS, and ISAISC methods.

\subsection{Evaluation Criteria}

The resultant images are evaluated qualitatively by user inspection. Furthermore, the quantitative evaluation is carried out using numerical metrics. The most prominent assessment criteria adopted for color images, when comparing performance among available methods, are colorfulness and saturation. Other common metrics for image quality measures in terms of brightness, information, gradient and contrast are also used.

\subsubsection{Colorfulness}

An image can be quantified by its colorfulness defined as [20]

$$
\mathcal{C}=\sigma_{\text {rgyb }}+0.3 \times \mu_{\text {rgyb }}
$$

where

$$
\sigma_{r g y b}=\sqrt{\sigma_{r g}^{2}+\sigma_{y b}^{2}}, \quad \mu_{r g y b}=\sqrt{\mu_{r g}^{2}+\mu_{y b}^{2}}
$$

It depends on the standard deviations $\sigma_{r g}, \sigma_{y b}$ of color differences of the enhanced color image, i.e., $\Delta_{r g}=R-G$ and $\Delta_{y b}=0.5(R+G)-B$. Furthermore, $\mu_{r g}, \mu_{y b}$ are the averages of $\Delta r g$ and $\Delta y b$. This measure will be small when an outdoor object is captured under bad weather conditions such as haze. A large colorfulness indicated an image for better viewing. 


\subsubsection{Saturation}

In addition to colorfulness, human perception also concerns with image saturation. This metric is adopted from the S-component of the hue-saturation-intensity color space [16]. The average saturation of all pixels used in the assessment is given as

$$
\mathcal{S}=\frac{1}{N} \sum S(u, v)
$$

where $N$ is the total number of pixels in the image, and $S(u, v)=1-\frac{\min \{R, G, B\}(u, v)}{\max \{R, G, B\}(u, v)}$ is the saturation of a pixel. Higher saturation denotes a more vivid image.

\subsubsection{Brightness Error}

The maintenance of the brightness of the output image with respect to the input is a desirable feature of enhancement operations. This metric is

$$
\mathcal{B}=\mu\left(I_{\text {enh }}\right)-\mu\left(I_{\text {in }}\right)
$$

where $\mu\left(I_{e n h}\right)$ is the global mean intensity of the enhanced image, $\mu\left(I_{i n}\right)$ is the global mean intensity of the input image. A brightness error at zero is an exact match of the input and output image brightness [21]. Furthermore, a positive or negative error could indicate a drift in brightness.

\subsubsection{Entropy}

One of the functions demanded from an image is to convey the scene information to the viewer. To this end, a popular measure is the information content or entropy given by

$$
\mathcal{H}=-\sum_{i=0}^{L-1} p(i) \log p(i)
$$

where $p(i)$ is the probability that a pixel has the intensity $i$. A high entropy value denotes desirable high information content carried in the image [22].

\subsubsection{Gradient}

Gradient is a simple measure that an object is detectable from the background as compared to its surrounding. It is defined as

$$
\mathcal{G}=\frac{1}{N} \sum_{(u, v) \in \Omega} \sqrt{\nabla_{u}^{2}(u, v)+\nabla_{v}^{2}(u, v)}
$$

where $N$ is the total number of pixels, $\Omega$ is the neighborhood of size $3 \times 3, \nabla_{u}(u, v)=I_{e n h}(u, v)-$ $I_{e n h}(u+1, v), \nabla_{v}(u, v)=I_{e n h}(u, v)-I_{e n h}(u, v+1)$ are the gradients along the horizontal and vertical directions across the image [16]. A high value quantifies a desirable sharp image. 
Journal of Modern Optics

\subsubsection{Contrast}

Contrast is another common measure of the sharpness of objects captured in the image. A larger value represents a higher contrast image [23]. The contrast is obtained from

$$
\mathcal{K}=\frac{1}{N} \sum_{(u, v)} I_{e n h}^{2}(u, v)-\left(\frac{1}{N} \sum_{(u, v)} I_{e n h}(u, v)\right)^{2} .
$$

\subsection{Qualitative Evaluation}

Two sample test images from the benchmark data set and two in-house captured real-world images are used as illustrations for qualitative viewing evaluation. Figures 2 and 3 show the enhancement results for the benchmark data set. Histogram based approaches including UNFHE, MMSICHE, and SMHEQ; in general, produce over-enhancements particularly at the ends of the intensity spectrum where regions in the output images are too dark or too bright. On the other hand, results from intensity and saturation adjustment approaches do not contain noticeable over-enhancement artifacts. In Figure 2, the outputs from the proposed approaches, ISAIS and ISAISC, have indicated the highlight of textures on the rocks in the near-end and the house in the far-end. In Figure 3, details of the grass at the near-end are enhanced.

Results from in-house collected test images are given in Figures 4 and 5. Similar observations are obtained as those found in test images 1 and 2. The developed methods in this work are able to provide satisfactory performances in the context of contrast and color vividness. Figures 4 and 5 both show that the details in the foreground plant and buildings in the background have been improved by the proposed ISAIS and ISAISC algorithms.

\subsection{Quantitative Evaluation}

In the quantitative evaluation, the set of performance metrics introduced in Section 4.1 are used. Statistics collected from the result images are assessed using box plots and hypothetical tests.

\subsubsection{Evaluation Criteria Box Plots}

The statistics of test results in colorfulness is given in Figure 6(a) as box plots. It can be seen that the colorfulness from the proposed ISAISC is the highest among all tested methods in the experiment, with a mean value of 0.095 . Other methods produce a measure of colorfulness across a range of 0.076 to 0.084 . This assessment indicator signifies that the correlated intensity and saturation adjustment method is very effective for color image contrast enhancement.

Figure 6(b) shows the box plot with regard to the saturation criterion. The saturation measurement obtained using the proposed ISAISC method has an average value of 0.116 , which is better than the ISAIS at 0.114. Furthermore, both methods perform much better than the other techniques evaluated.

The brightness error box plot between the input and enhanced images are depicted in Figure 6(c). Both proposed methods have an error close to 0.000. Results measured against the information content, entropy, are given in Figure 6(d). It can be seen that entropy values obtained from the developed ISAISC method is the highest among all tested techniques, with a mean value of 7.454. Note that other methods produce a measure of entropy across a range of 7.141 to 7.445. On the other hand, lower entropies are observed from UNFHE, MMSICHE, and SMHEQ.

Figure 6(e) illustrates the image gradients of the compared methods. The mean gradient of the results from the proposed ISAISC is 0.043 , compromising the gain in saturation and comparable to ISAIS at 0.044 , but is better than other techniques begin compared. Measured results of the contrast criterion are consolidated into the box plot in Figure 6(f). The contrast measurements 


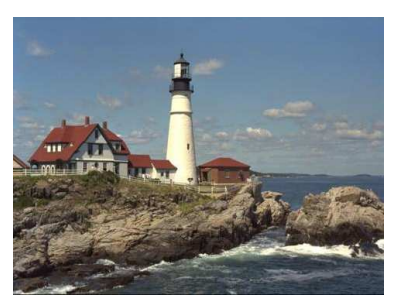

(a)

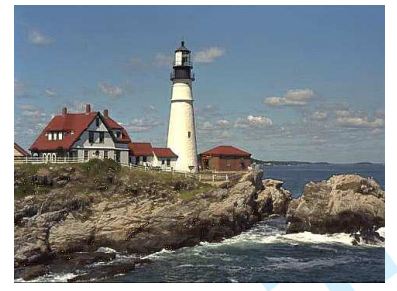

(e)

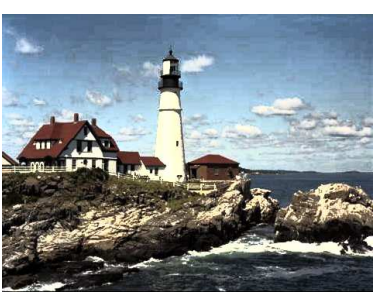

(b)

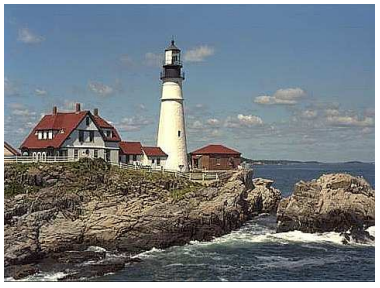

(f)

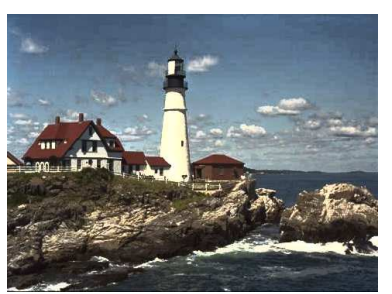

(c)

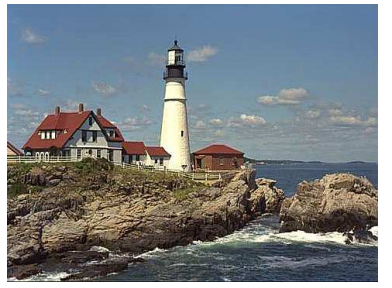

(g)

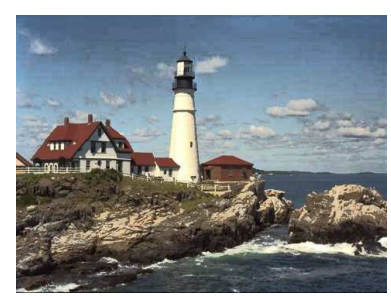

(d)

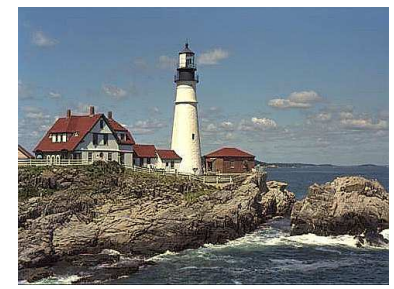

(h)

Figure 2. Enhancement results - Test image 1: (a) Input, (b) UNFHE, (c) MMSICHE, (d) SMHEQ, (e) IAIS, (f) IAISC, (g) ISAIS, (h) ISAISC.

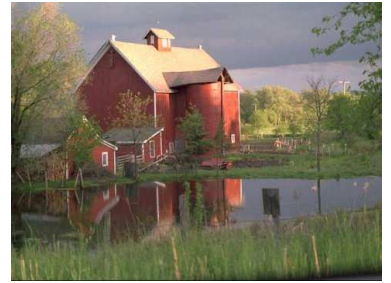

(a)

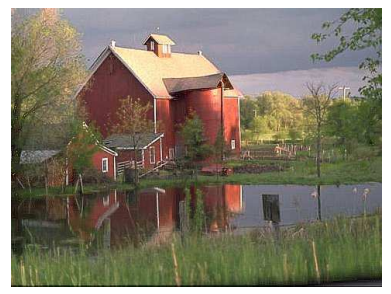

(e)

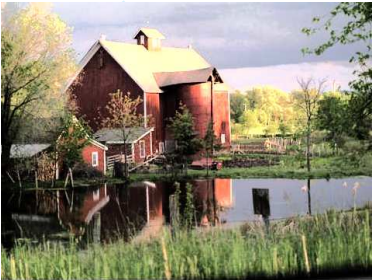

(b)

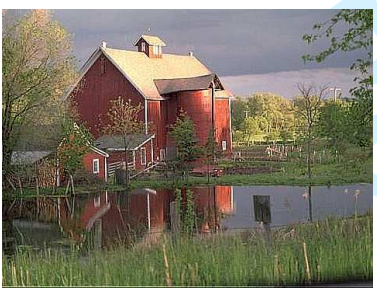

(f)

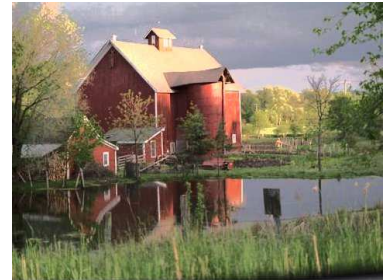

(c)

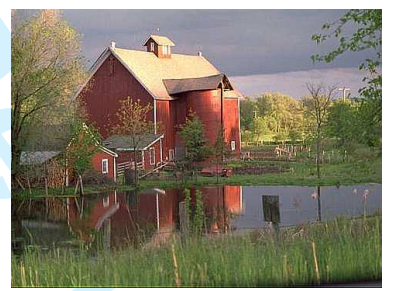

(g)

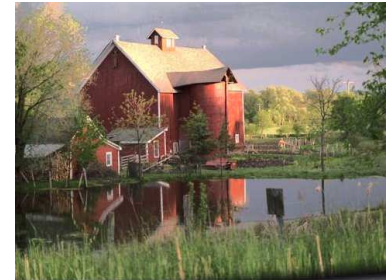

(d)

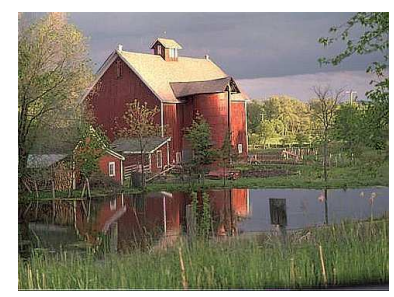

(h)

Figure 3. Enhancement results - Test image 2: (a) Input, (b) UNFHE, (c) MMSICHE, (d) SMHEQ, (e) IAIS, (f) IAISC, (g) ISAIS, (h) ISAISC.

obtained by using the proposed method have a mean value of 0.559. Although not the highest, improvement over the input image is obtained.

\subsubsection{Hypothetical Tests}

The paired Student's t-test was used to check if the distribution of the mean of the output image metric is the same as the input for a 0.05 significance level. The hypotheses are:

$$
\begin{aligned}
& H_{0} \text { : the means of input-output metrics are equal, } \\
& H_{1} \text { : the means of input-output metrics are not equal. }
\end{aligned}
$$

Test results, sorted in ascending magnitude for better visualization, are shown in Figure 7 for the metrics of the proposed ISAISC as compared to the input and other methods. For colorfulness, Figure 7(a), the null hypothesis is rejected. By observing the mean values, it can be concluded that ISAISC outperforms the other methods. Figure 7(b) shows the saturation metric and the null hypothesis is rejected. The average value of this metric also proves that ISAISC is effective with 


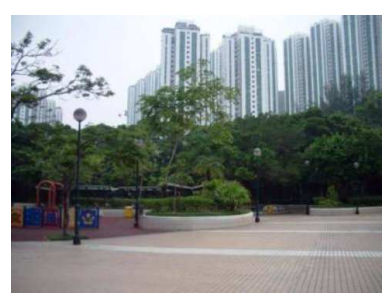

(a)

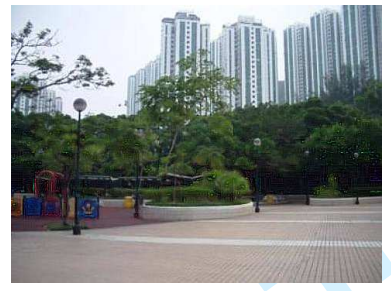

(e)

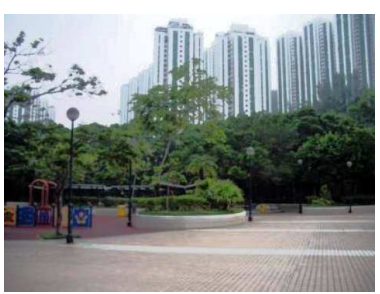

(b)

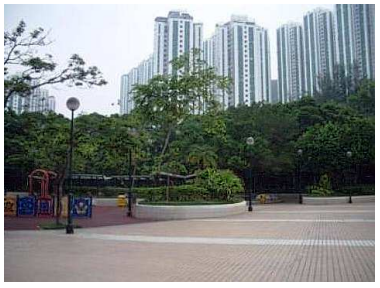

(f)

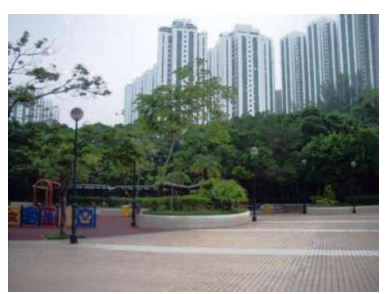

(c)

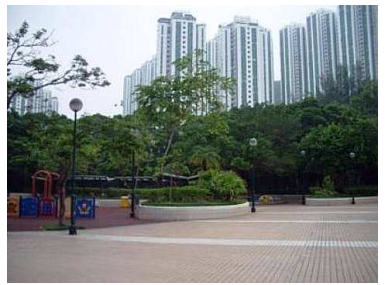

(g)

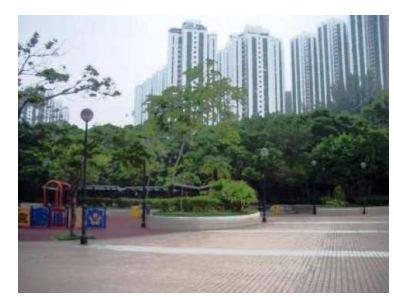

(d)

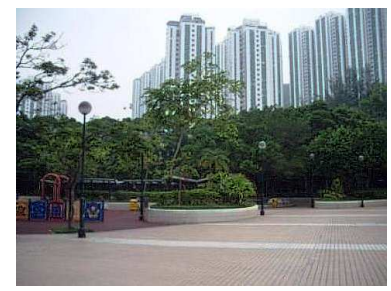

(h)

Figure 4. Enhancement results - Test image 3: (a) Input, (b) UNFHE, (c) MMSICHE, (d) SMHEQ, (e) IAIS, (f) IAISC, (g) ISAIS, (h) ISAISC.

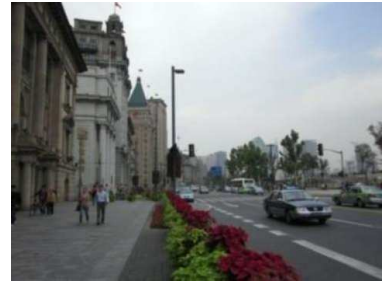

(a)

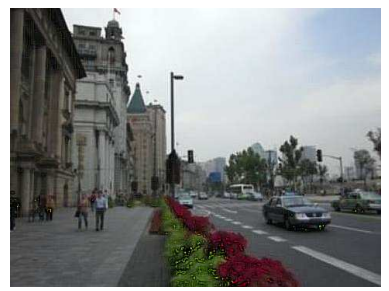

(e)

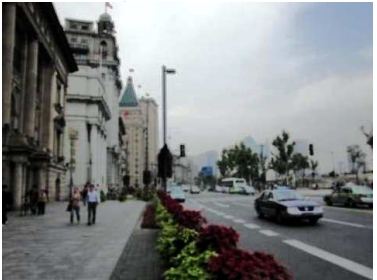

(b)

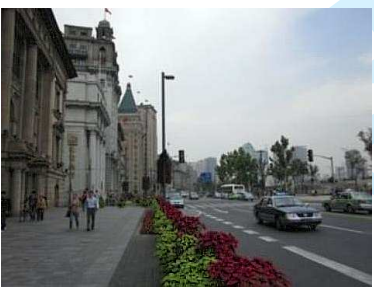

(f)

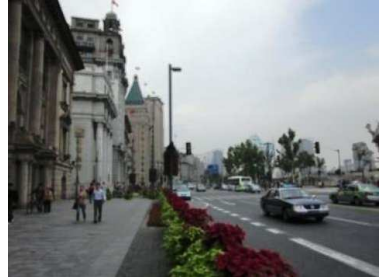

(c)

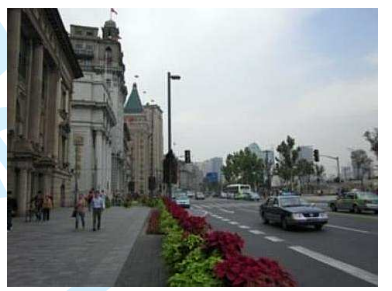

(g)

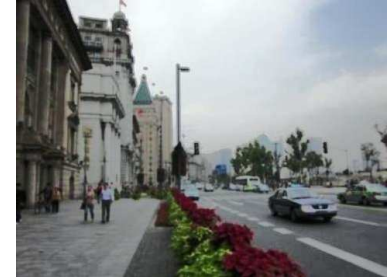

(d)

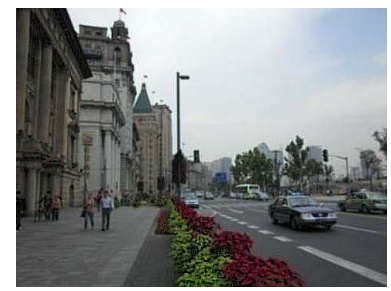

(h)

Figure 5. Enhancement results - Test image 4: (a) Input, (b) UNFHE, (c) MMSICHE, (d) SMHEQ, (e) IAIS, (f) IAISC, (g) ISAIS, (h) ISAISC.

regard to saturation enhancement. For the brightness error, the null hypothesis is not rejected, see Figure $7(\mathrm{c})$. This indicates that the proposed algorithm is able to preserve the brightness of the input image.

The test for output image information content or entropy shows that the null hypothesis is rejected, Figure 7(d). Together with the average entropy of the test set, the ISAIC method is able to produce an increased entropy output. The test results for gradient are depicted in Figure 7(e), the null hypothesis is rejected with an extremely small p-value denoting an increase in gradient. Test statistics for contrast is shown in Figure $7(\mathrm{f})$, the null hypothesis is not rejected for ISAISC. This metric is also not rejected in other methods except for the SMHEQ.

\subsection{Discussion}

Enhancing the quality of color images has to be considered for improving both the contrast and saturation in order to make the image more content informative and color vivid. As an extension to the conventional enhancement approaches, which focuses on improving only the image contrast, 


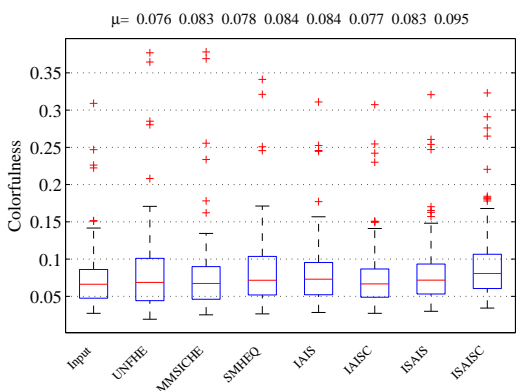

(a)

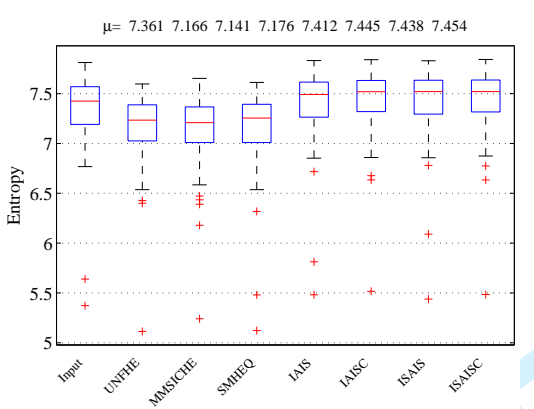

(d)

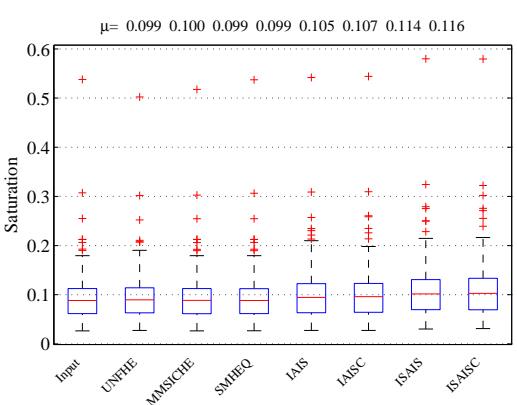

(b)

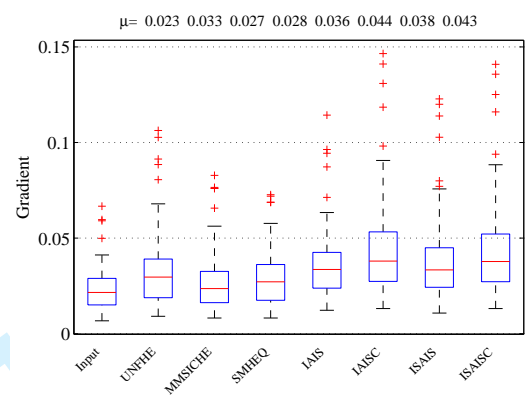

(e)

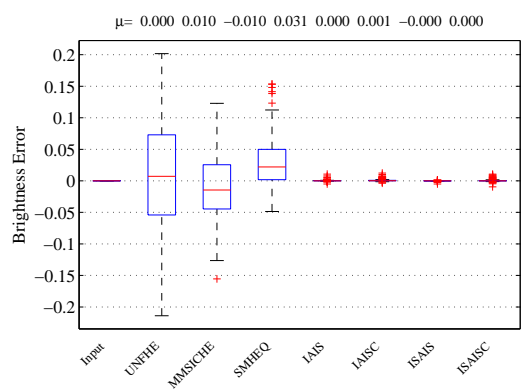

(c)

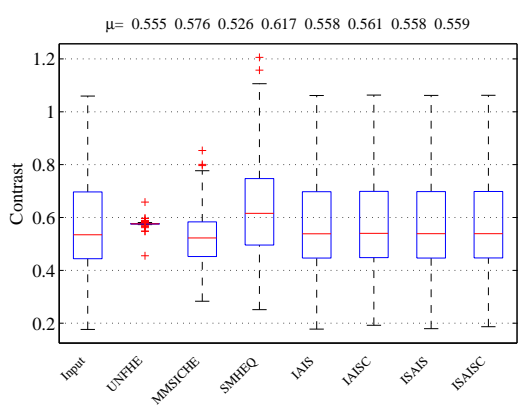

(f)

Figure 6. Result statistics - box plots: (a) colorfulness, (b) saturation, (c) brightness, (d) entropy, (e) gradient, (f) contrast.

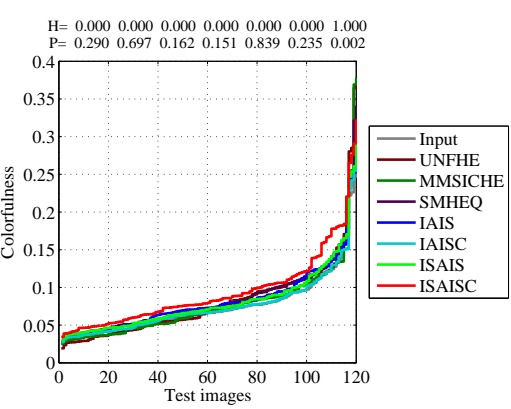

(a)

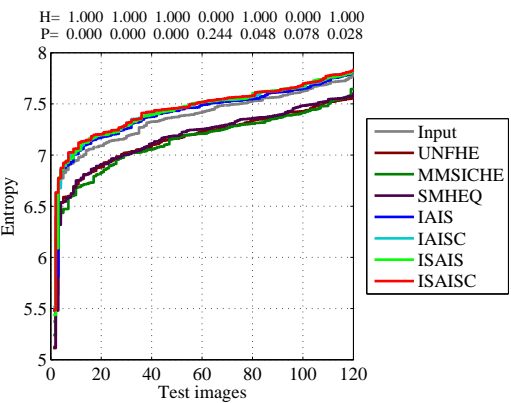

(d)

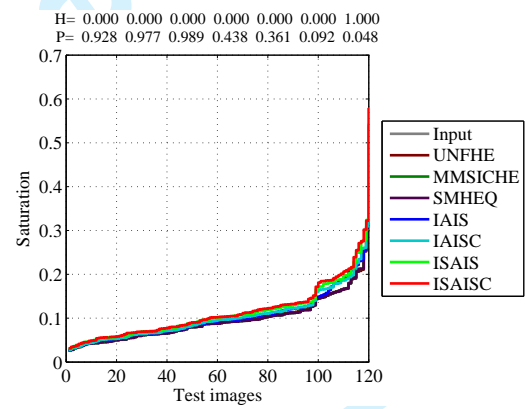

(b)

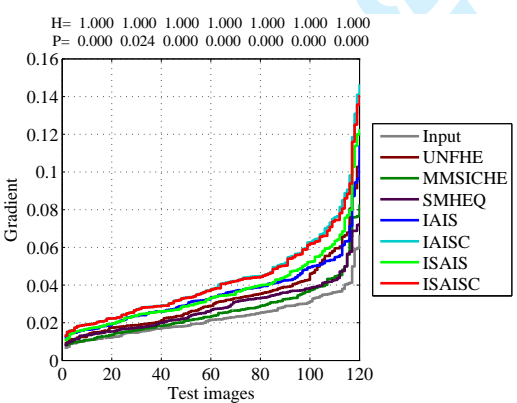

(e)

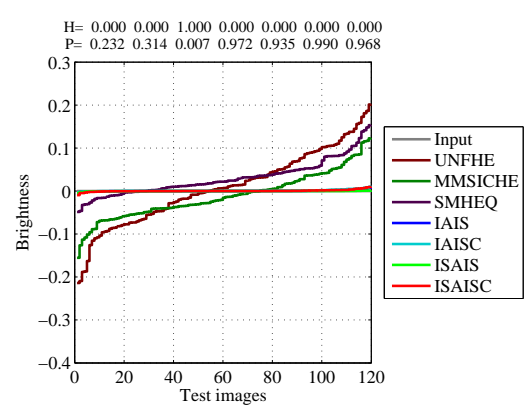

(c)

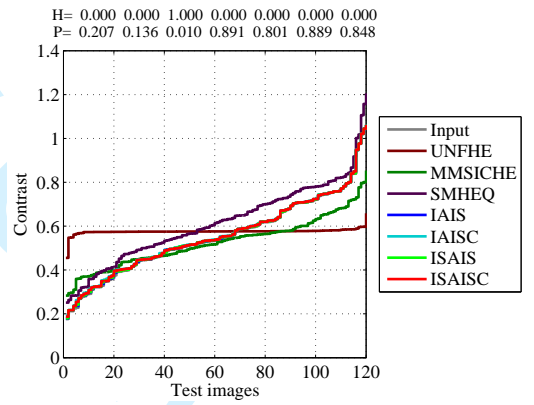

(f)

Figure 7. Result statistics: (a) colorfulness, (b) saturation, (c) brightness, (d) entropy, (e) gradient, (f) contrast.

the problem of simultaneous increases in contrast and saturation is addressed in this work. From the qualitative and quantitative evaluations, it can be concluded that the proposed ISAIS and ISAISC approaches both perform well as proven by qualitative viewing and quantitative metrics. It should be noted that the ISAIS method, a simplified algorithm without the need to calculate the correlation value, is performing similarly to the ISAISC. However, the latter although performs better, needs to include the time consuming calculation of the correlation. The performance of 
the ISAIS approach, as can be observed, is comparable to ISAISC where the former required less computations.

\section{Conclusion}

This paper has presented the development of algorithms for color image quality enhancement, in the context of contrast and color vividness, using intensity and saturation adjustments. It is found that simultaneous modifications on both intensity and saturation are useful in improving the quality of color images. The proposed algorithms contain a lattice structure making better use of intensity, saturation and their correlation to enhance the image. In addition to improvements in contrast and color vividness, the proposed method is also able to maintain the output image global brightness as compared to the input. Furthermore, as indicated by the experiment results, it is suggested that a compromise might have to be considered between the computational efficiency and enhancement effectiveness according to the application domain.

\section{References}

[1] W. Guo, X. Xia, and W. Xiaofei, "A remote sensing ship recognition method based on dynamic probability generative model," Expert Systems with Applications, vol. 41, no. 14, pp. 6446-6458, 2014.

[2] T. Wu, H. Wu, Y. Du, N. Kwok, and Z. Peng, "Imaged wear debris separation for on-line monitoring using gray level and integrated morphological features," Wear, vol. 316, no. 1-2, pp. 19-29, 2014.

[3] S.-C. Pei and Y.-M. Chiu, "Background adjustment and saturation enhancement in ancient chinese paintings," Image Processing, IEEE Transactions on, vol. 15, no. 10, pp. 3230-3234, Oct 2006.

[4] Y.-H. Yu, N. M. Kwok, and Q. P. Ha, "Color tracking for multiple robot control using a system-onprogrammable- chip," Automation in Construction, vol. 20, no. 6, pp. 669-676, 2011.

[5] N. M. Kwok, H. Y. Shi, Q. P. Ha, G. Fang, S. Y. Chen, and X. Jia, "Simultaneous image color correction and enhancement using particle swarm optimization," Engineering Applications of Artificial Intelligence, vol. 26, no. 10, pp. 2356-2371, 2013.

[6] G. Jiang, C. Wong, S. Lin, M. Rahman, T. Ren, N. Kwok, H. Shi, Y.-H. Yu, and T. Wu, "Image contrast enhancement with brightness preservation using an optimal gamma correction and weighted sum approach," Journal of Modern Optics, pp. 1-12, 2015.

[7] K. Sagawa, "Visual comfort to colored images evaluated by saturation distribution," Color Research and Application, vol. 24, no. 5, pp. 313-321, 1999.

[8] L. Lucchese, S. Mitra, and J. Mukherjee, "A new algorithm based on saturation and desaturation in the xy chromaticity diagram for enhancement and re-rendition of color images," in Image Processing, 2001. Proceedings. 2001 International Conference on, vol. 2, Oct 2001, pp. 1077-1080 vol.2.

[9] J.-Y. You and S.-I. Chien, "Saturation enhancement of blue sky for increasing preference of scenery images," Consumer Electronics, IEEE Transactions on, vol. 54, no. 2, pp. 762-768, May 2008.

[10] V. S. Bhadouria and D. Ghoshal, "A study on computationally fast colour image enhancement based on fuzzification of saturation and intensity components," International Journal of Innovative Computing and Applications, vol. 4, no. 2, pp. 67-74, 2012.

[11] R. N. Strickland, C.-S. Kim, and W. F. McDonnell, "Digital color image enhancement based on the saturation component," Optical Engineering, vol. 26, no. 7, pp. 267609-267609-, 1987.

[12] B. Thomas, R. Strickland, and J. Rodriguez, "Color image enhancement using spatially adaptive saturation feedback," in Image Processing, 1997. Proceedings., International Conference on, vol. 3, Oct 1997, pp. 30-33 vol.3.

[13] G. Song and X.-l. Qiao, "Color image enhancement based on luminance and saturation components," in Image and Signal Processing, 2008. CISP '08. Congress on, vol. 3, May 2008, pp. 307-310.

[14] C.-C. Yang, "Improving the sharpness of a color image with slowly-varying hues by undertaking its intensity and saturation factors," Optik - International Journal for Light and Electron Optics, vol. 125, no. 22 , pp. $6730-6732,2014$.

[15] K. Nakai, Y. Hoshi, and A. Taguchi, "Color image contrast enhacement method based on differen- 
tial intensity/saturation gray-levels histograms," in Intelligent Signal Processing and Communications Systems (ISPACS), 2013 International Symposium on, Nov 2013, pp. 445-449.

[16] R. C. Gonzalez and R. E. Woods, Digital Image Processing (3rd Edition). Upper Saddle River, NJ, USA: Prentice-Hall, Inc., 2006.

[17] N. M. Kwok, H. Y. Shi, G. Fang, and Q. P. Ha, "Intensity-based gain adaptive unsharp masking for image contrast enhancement," in 2012 5th International Congress on Image and Signal Processing, CISP 2012, 2012, pp. 529-533.

[18] K. Singh and R. Kapoor, "Image enhancement via median-mean based sub-image-clipped histogram equalization," Optik-International Journal for Light and Electron Optics, vol. 125, no. 17, pp. 46464651, 2014.

[19] N. M. Kwok, X. Jia, D. Wang, S. Y. Chen, G. Fang, and Q. P. Ha, "Visual impact enhancement via image histogram smoothing and continuous intensity relocation," Computers and Electrical Engineering, vol. 37, no. 5, pp. 681-694, 2011.

[20] D. Hasler and S. E. Suesstrunk, "Measuring colorfulness in natural images," in Electronic Imaging 2003. International Society for Optics and Photonics, 2003, pp. 87-95.

[21] Y.-T. Kim, "Contrast enhancement using brightness preserving bi-histogram equalization," Consumer Electronics, IEEE Transactions on, vol. 43, no. 1, pp. 1-8, 1997.

[22] Y. Yao, B. Abidi, N. Doggaz, and M. Abidi, "Evaluation of sharpness measures and search algorithms for the auto focusing of high-magnification images," in Defense and Security Symposium. International Society for Optics and Photonics, 2006, pp. 62 460G-62460G.

[23] J. R. Tang and N. A. M. Isa, "Adaptive image enhancement based on bi-histogram equalization with a clipping limit," Computers and Electrical Engineering, vol. 40, no. 8, pp. 86 - 103, 2014. 